\title{
Alveolar Bone Resorption Evaluation Around Single-piece Designed Bicortical Implants, Using Immediate Loading Protocol, Based on Orthopantomographs
}

\author{
Dániel-Tamás Száva1, Alina Ormenișan¹, Emese Markovics², Bálint Bögözi¹, Krisztina Mártha³ \\ 1 Department of Oral and Maxillofacial surgery, Faculty of Dental Medicine, University of Medicine and Pharmacy, Tîrgu Mureș, Romania \\ 2 Department of Removable Prosthetic Dentistry, Faculty of Dental Medicine, University of Medicine and Pharmacy, Tîrgu Mureș, Romania \\ 3 Department of Orthodontics, Faculty of Dental Medicine, University of Medicine and Pharmacy, Tîrgu Mureș, Romania
}

\section{CORRESPONDENCE \\ Dániel-Tamás Száva \\ Str. Bolyai nr. 16 \\ 540056 Tîrgu Mureș, Romania \\ Tel: +40 747966136 \\ E-mail: szavadani@yahoo.com}

\section{ARTICLE HISTORY}

Received: December 13, 2017

Accepted: December 29, 2017
Alina Ormenișan • Str. Gheorghe Marinescu nr. 38 540139 Tîrgu Mureș, Romania. Tel: +40 265215551 Emese Markovics • Str. Gheorghe Marinescu nr. 38 , 540139 Tîrgu Mureș, Romania. Tel: +40 265215551 Bálint Bögözi • Str. Gheorghe Marinescu nr. 38 , 540139 Tîrgu Mureș, Romania. Tel: +40 265215551 Krisztina Mártha • Str. Gheorghe Marinescu nr. 38 540139 Tîrgu Mures, Romania. Tel: +40 265215551

\begin{abstract}
Background: Inserting dental implants in severely atrophied jawbones is a great challenge for the dental practitioner. There are an increasing number of patients who choose dental implantanchored prosthetic restorations despite compromised bone quality and quantity. There have been numerous attempts in adapting implant design for the atrophic crestal bone. One-piece, needle-type basal implant design is a typical design for these cases. These implants are inserted in the remaining compact bone located in the basal aspect of the jawbones. If high primary stability is achieved, these implants are used for immediate loading protocol. From many points of view, this technique is based on contradictory principles compared to classic implant surgery and loading protocols. The aim of this study was to investigate the long-term success of basal one-piece short-diameter dental implants used for immediate loading protocol. Materials and methods: A total of 56 dental implants were included in this study. Peri-implant bone loss was measured on orthopantomographs. Bone resorption was measured in millimeters in the first 6 and 12 months of functional loading. Results: There were no failing implants in this period; average bone resorption was $1.59 \mathrm{~mm}$ after 6 months of functional loading and 2.05 mm after 12 months. Bone resorption was slightly higher in the mandible than in the maxilla. Conclusions: Immediate implant loading protocol might be an attractive solution for fixed fullarch restoration using short-diameter one-stage basal implants, but long-term effects require further investigations.
\end{abstract}

Keywords: severe alveolar bone atrophy, small-diameter needle-type bicortical dental implant, immediate implant loading protocol

\section{INTRODUCTION}

Immediate implant loading protocol is getting widely adopted by dental practitioners due to the several advantages it offers. Firstly, it saves time. There is no healing period for 3 to 6 months, after which final restoration can be placed. With special 


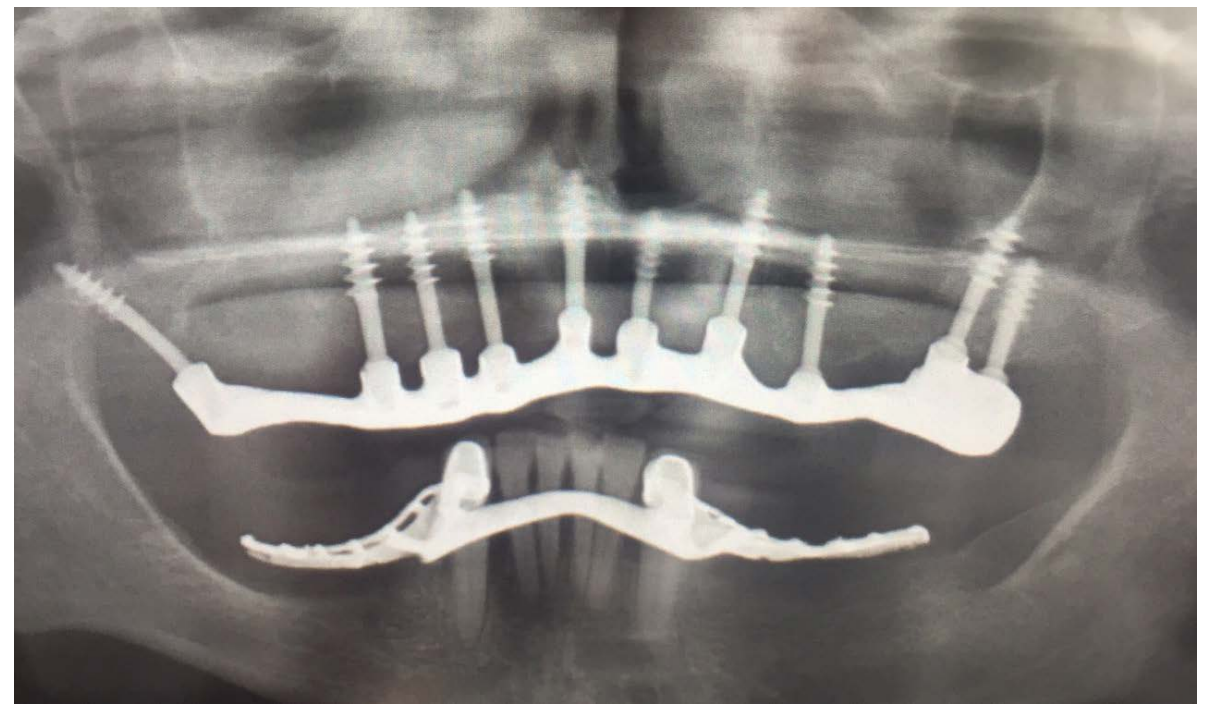

FIGURE 1. Orthopantomography at 6 months after implant placement

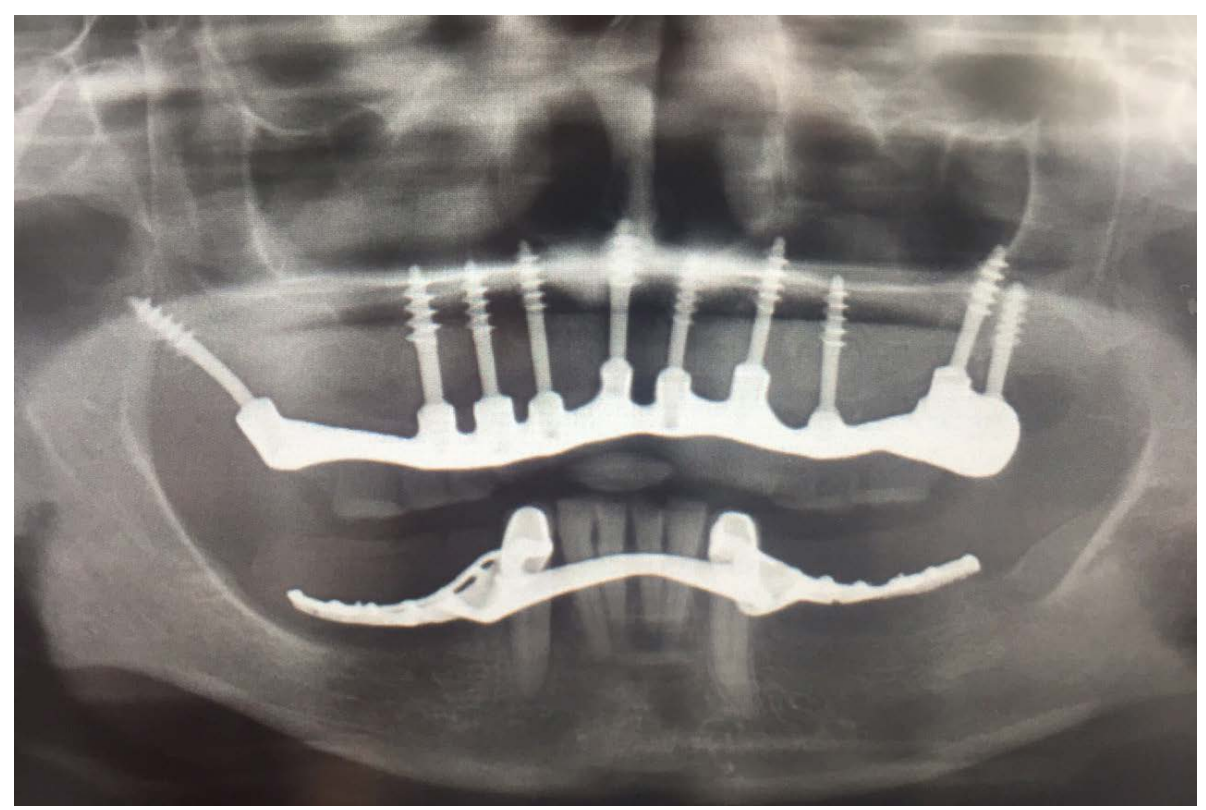

FIGURE 2. Orthopantomography at 12 months after implant placement

implant design, producers try also to eliminate bone augmentation procedures that could delay the final outcome of the dental restoration. Compared to two-piece dental implants, there is no second-stage operation required with all its inconveniences for the patient. ${ }^{1}$ Secondly, during the second surgery, in case of two-stage dental implants, the biological width formation leads to certain amount of bone loss. ${ }^{2}$ In the region of the second surgery, soft tissue height and width may modify, compromising esthetics in the frontal areas. Soft tissue-bone-supported temporary restoration applied for the healing period in case of two- stage dental implants can affect osteointegration of the underlying den- tal implants. In case of immediately loaded dental implants, splinting should be made within 2 weeks. Splinting the implants is superior from a biomechanical point of view compared to independent dental implant loading. ${ }^{1}$

One-piece dental implants also have some advantages compared to two-piece dental implants. There is no microgap formation between the implant platform and abutment, consequent vertical bone loss is lesser than in case of the two-stage dental implants, and crestal bone resorption may also be slower. ${ }^{3}$ It is also known that basal bone resorption is last to begin in the jawbones due to high bone density and continuous muscle stimulation. ${ }^{4}$ 

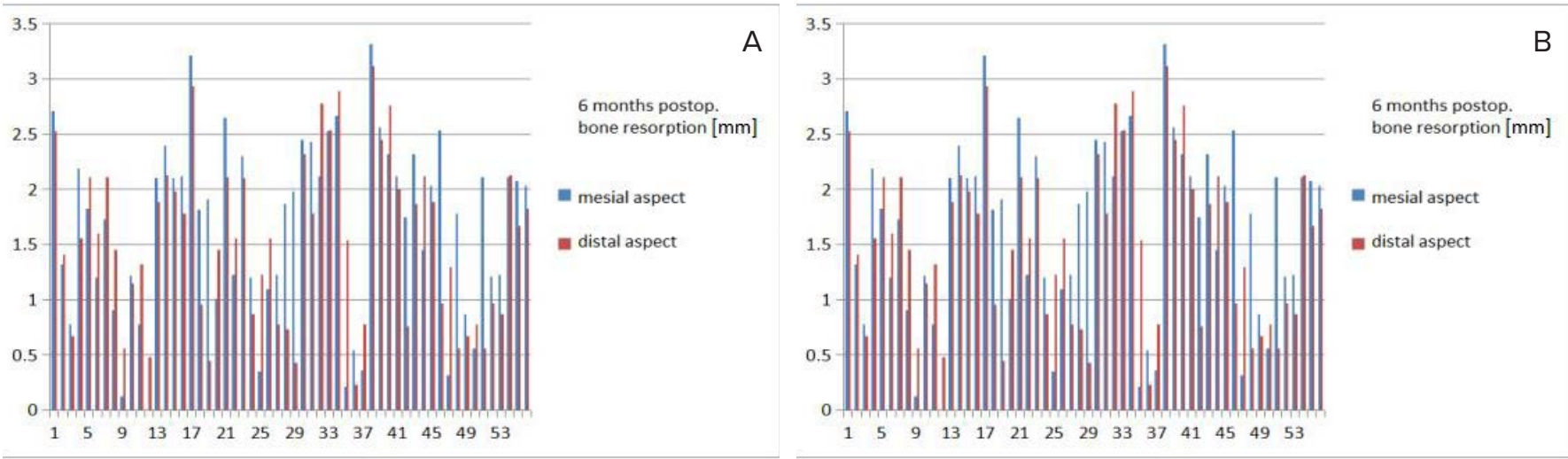

FIGURE 3. A - Postoperative bone resorption at 6 months after immediate loading. B - Postoperative bone resorption at 12 months after immediate loading.

Based on these principles, an innovative implant system was developed, which allows immediate implant insertion and loading protocol. The Ihde implant system ${ }^{5}$ (Dr. Ihde Dental AG, Dorfplatz 118737 Gommiswald, Switzerland) has developed one-piece basal implants. Some of its major characteristics are large thread depth, which increases primary stability, and large surface area, thus optimizing osteointegration. High implant stability reduces micromovement, with subsequent enabling of immediate loading.

The aim of our study was to compare alveolar bone resorption occurring near one-piece basal dental implants using immediate loading protocol with that occurring near two-piece designed implants, loaded after osteointegration was confirmed on retroalveolar radiographs.

\section{MATERIALS AND METHOD}

A total number of 56 basal, one-piece dental implants placed on 10 patients with immediate loading protocol were examined. The patients included in the study presented no general health issues. All patients were treated in the same dental office, and the implants were inserted by the same dental practitioner, using KOS- and BCS-type implants (Dr. Ihde Dental AG, Dorfplatz 118737 Gommiswald, Switzerland). Peri-implant bone resorption was measured on digital orthopantomographs (OPG) in the moment of placement and 6 and 12 months after functional loading (Figure 1 and Figure 2). On each radiograph, the vertical dimension of peri-implant bone quantity was measured in pixels, related to the known implant length.

\section{RESULTS}

The vertical mesial and distal peri-implant bone resorption measurements are presented in Figure 3.
Average bone resorption at 6 months following the procedure was $1.66 \mathrm{~mm}$ mesial and $1.53 \mathrm{~mm}$ distal to the implant surface. The average bone resorption at 12 months was $2.1 \mathrm{~mm}$ on the mesial region and $2.01 \mathrm{~mm}$ on the distal surface (Figure 4). Average bone resorption around singletooth implant restorations was $2.18 \mathrm{~mm}$, whereas around splinted implants the measured vertical bone resorption was $1.99 \mathrm{~mm}$.

\section{DISCUSSIONS}

Implantology is characterized by innovation and openness to new solutions. Bone quantity and quality may be the most important factor that restricts dental implant insertion. There are several methods applied for bone augmentation, which can delay the immediate implant loading protocol. In order to eliminate bone augmentation procedures in case of severely atrophied jawbones, specially designed dental implants were invented, namely

AVERAGE BONE RESORPTION [MM]

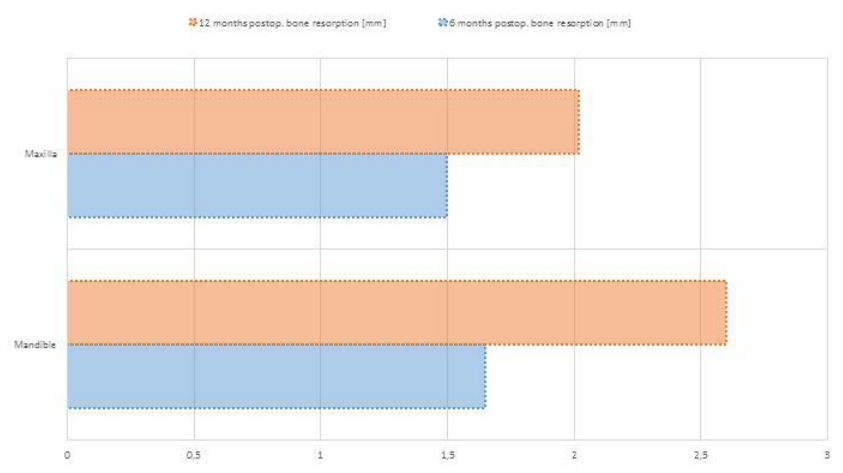

FIGURE 4. Average bone resorption at 6 and 12 months after the procedure 
bicortical needle implants. Bicortical insertion offers high primary stability, enabling immediate restoration placement. Short-term advantages represent attractive properties of these implants. Some studies suggest that implant design and thread depth may not be the most important factors that influence the short-term success rate of immediate implant loading. Implants within the 3-6 weeks postoperative period are most susceptible to loading failure due to bone remodeling of the peri-implant surgical area. ${ }^{6}$ The number of implants and the type of prosthetic restorations can also influence the success rate of dental implants. Some studies showed that immediately loaded one-stage implants used for single-tooth restorations showed a $99 \%$ success rate. ${ }^{7,8}$

In case of supported multiple dental implants, the longterm success rate varies between $92.8 \%$ and $97.5 \% .^{9}$

In the particular case of implants for full arch restorations supported by an edentulous jaw, carried out with immediate implant loading protocol, splinting the dental implants favors the overall survival rate. ${ }^{10}$ In this study about specially designed basal implants used for immediate loading protocol no implant failures were reported, and the short-term success rate was $100 \%$.

We found it important to investigate peri-implant bone loss for the evaluation of long-term success of these implants.

Average bone loss after functional loading was $1.59 \mathrm{~mm}$ at 6 months and $2.05 \mathrm{~mm}$ after 12 months. These values correspond with those of other authors who found 2-3 mm of bone loss after 1 year of loading. ${ }^{11}$

We also studied how splinting influenced alveolar bone resorption and found that bone resorption was higher near single-tooth implants $(2.18 \mathrm{~mm})$ than in case of multiple, splinted implants $(1.99 \mathrm{~mm})$. This proves that the number and position of the implant, as well as the type of dental restoration are the main factors that influence long-term success.

Finally, average bone resorption in case of one-stage, immediately loaded basal implants was compared to that of two-stage implants loaded after osteointegration. After the first year, the two-stage, osteointegrated implants showed less bone resorption $(1.25 \mathrm{~mm})$ than the immediately-loaded one-stage basal implants $(2.05 \mathrm{~mm})$. In case of our study population, we must consider that basal implants were inserted in severely atrophied jawbones, where the vertical occlusal dimension has probably grown, thus creating a less favorable situation and potentially overloading the dental implants.

\section{CONCLUSIONS}

Average bone resorption in the first 12 months of functional loading was less than $2.5 \mathrm{~mm}$ in the studied cases, as usually mentioned in the literature. Splinted implants showed less nearby bone resorption than single-tooth restorations. Implants placed in the maxilla showed less bone loss than implants placed in the mandible. Significantly higher bone loss was measured in the first 12 months of functional loading than in case of two-stage dental implants. Immediate implant loading protocol might be an attractive solution for fixed full arch restoration using short-diameter one-stage basal implants, but long-term effects require further investigations.

\section{CONFLICT OF INTEREST}

Nothing to declare.

\section{REFERENCES}

1. Misch CE. Contemporary Implant Dentistry, Third Edition. St. Louis: Mosby, 2008; p. 833

2. Szava D. Cercetări experimentale privind biomecanica implanturilor dentare prin metoda Video Image Correlation. Doctoral thesis, University of Medicine and Pharmacy of Tîrgu Mures, 2015.

3. Misch CE. Early crestal bone loss etiology and its effect on treatment planning for implants. Postgrad Dent. 1995;2:3-17.

4. Atwood DA, Coy WA. Clinical cephalometric and densitometric study of reduction of residual ridges. J Prosthet Dent. 1971;26:280-295.

5. Ihde S. Principles of BOI. Berlin, Heidelberg: Springer-Verlag, 2004; p. 2530

6. Buchs AU, Levine L, Moy P. Preliminary report of immediately loaded Altiva natural tooth replacement dental implants. Clin Implant Dent Relat Res. 2001;3:97-105.

7. Kan JY, Rungcharassaeng K. Immediate implant placement and provisionalization of maxillary anterior single implants: a surgical and prosthodontic rationale. Pract Periodont Aesthet Dent. 2000;12:817-824.

8. Choquet $V$, Hermans $M$, Adriaenssens $P$, et al. Clinical and radiographic evaluation of the papilla level adjacent to single tooth dental implants: a retrospective study in the maxillary anterior region. J Periodontol. 2001;72:1364-1371.

9. Kline R, Hoar J, Beck GH. A prospective multicenter clinical investigation of a bone quality-based dental implant system. Implant Dent. 2002;11:1-8.

10. Misch CE, Wang $\mathrm{H}-\mathrm{L}$, Misch $\mathrm{CM}$, et al. Rationale for the application of immediate load in implant dentistry: part II. Implant Dent. 2004;13:310-321.

11. Werner M, Thomas F. Implantológia külföldön. Available at: http://www. sofortimplant.hu/download/artikel/azonnali-terheles-cirkularis-kosrekonstrukciok-hosszutavu-vizsgalata-kopie.pdf. 2012 